\title{
A Dog with Osteosarcoma which Metastasized to the Eye Months before Metastasis to Other Organs
}

\author{
Hiroto YOSHIKAWA ${ }^{1,2)}$, Yuya NAKAMOTO ${ }^{2)}$, Tsuyoshi OZAWA ${ }^{2)}$ and Ryan M. DICKINSON ${ }^{3)}$ \\ ${ }^{1)}$ Department of Small Animal Clinical Sciences, Western College of Veterinary Medicine, University of Saskatchewan, 52 Campus Drive, \\ Saskatoon, Saskatchewan, S7N 5B4, Canada, ${ }^{2}$ Ozawa Animal Hospital, 4, Niban-Uji, Uji City, Kyoto, 611-0021, Japan and \\ ${ }^{3)}$ Department of Pathology, Western College of Veterinary Medicine, University of Saskatchewan, 52 Campus Drive, Saskatoon, \\ Saskatchewan, S7N 5B4, Canada
}

(Received 27 November 2007/Accepted 10 April 2008)

ABSTRACT. A 9-year-old male Shih Tzu with osteosarcoma had a forelimb amputation and underwent chemotherapy. During chemotheapy, the right eye was enucleated due to refractory glaucoma, and was diagnosed as anterior uveal malignant melanoma. The dog lived for 4 months after the enucleation without treatment. After the dog died, the mass in the eye was re-evaluated immunohistochemically, and it was diagnosed as metastasis of appendicular osteosarcoma. Metastasis of appendicular osteosarcoma to the anterior chamber is quite rare, and the clinical course which showed clinically detectable metastases to the eye before systemic multi-organ metastases was quite unique.

KEY WORDS: canine, metastasis to the eye, osteosarcoma.

Appendicular osteosarcoma in dogs is the most common bone tumor, especially in large breed dogs, and it is highly metastatic [3]. Amputation of the affected limb is usually recommended because of severe pain and high risk of pathological fracture, although amputation alone can't extend survival time [3]. Extension of survival time by postoperative systemic chemotherapy is proven in many reports; however, almost all patients die due to distant metastasis [3]. On this occasion, we report a dog with appendicular osteosarcoma treated by amputation and postoperative systemic chemotherapy, which metastasized to the anterior uveal chamber several months before detection of multi-organ metastases. Such clinical course hasn't been reported previously and is thought to be very rare.

The dog in this report was a 9-year-old intact male Shih Tzu, weighing $5.4 \mathrm{~kg}$. Histopathologically, this dog was diagnosed at Ozawa Animal Hospital as having osteosarcoma in the left proximal humerus. No abnormalities were found on three-view thoracic radiographs. Consequently, the dog's leg was amputated.

Histopathological examination of the leg revealed tumor cells with marked anisokaryosis, oval to round nuclei with prominent and multiple nucleoli, and eosinophilic cytoplasm had been proliferating diffusely or solidly and infiltrating into existing bone tissues (Fig. 1). Osteoid production was also seen. Considering these findings, appendicular osteosarcoma was diagnosed.

On day 14 following surgery, chemotherapy with carboplatin at a dose of $300 \mathrm{mg} / \mathrm{m}^{2}$ was started and repeated 6 times with 18 to 23 day intervals. During this therapy, no

\footnotetext{
* Correspondence to: Yoshikawa, H., Small Animal Clinical Sciences, Veterinary Teaching Hospital, Western College of Veterinary Medicine, University of Saskatchewan, Saskatoon, SK S7N5B4, Canada.

e-mail: hiroto.yoshikawa@usask.ca
}

abnormalities were detected. On day 98 following the first presentation, which was day 9 following the 5 th chemotherapy treatment, purulent discharge and slight conjunctivitis was noted in the right eye and was treated with antibacterial eye drops, because bacterial conjunctivitis was suspected. On day 107, intraocular pressure (IOP) was measured and found to be within normal limits (average 16.5 $\mathrm{mmHg}$, normal limit is less than $25 \mathrm{mmHg}$ ). During the 6th chemotherapy treatment on day 111, the conjunctivitis of the right eye was slightly improved, but hyphaema and pain in the eye were noted. On day 118, increased IOP (average $42 \mathrm{mmHg}$ ) was noted, and a small, demarcated pale mass was found on the iris of the right eye. At this time, secondary glaucoma was suspected, and treatments with mannitol ( $2 \mathrm{~g} / \mathrm{kg}$ i.v. over $15 \mathrm{~min}$ ), latanoprost eye drops (1drop q24h to the right eye), and methazolamide $(2.5 \mathrm{mg} / \mathrm{kg}$, p.o bid) were started and latanoprost and methazolamide were continued until day 142 without improvement. Enucleation of the right eye was performed due to the blindness in the right eye. No other lesions were found by three-view thoracic radiographs and other examinations. On histopathological examination, at this time, the tumor was diagnosed as anterior uveal malignant melanoma. After the postoperative period, we suggested re-starting chemotherapy to the owner, but this was declined due to financial reasons. All medications were discontinued.

On day 237, the dog was presented because of slight anorexia. Rhonchus was heard by auscultation, and many nodules which strongly suggested lung metastases were revealed radiographically. On day 248 , nodules with a mixed echogenic pattern were detected on the left kidney and spleen by abdominal ultrasound examination. On day 272, the dog died at his home. Postmortem examination was not performed.

Paraffin embedded biopsy samples of the tumor were 

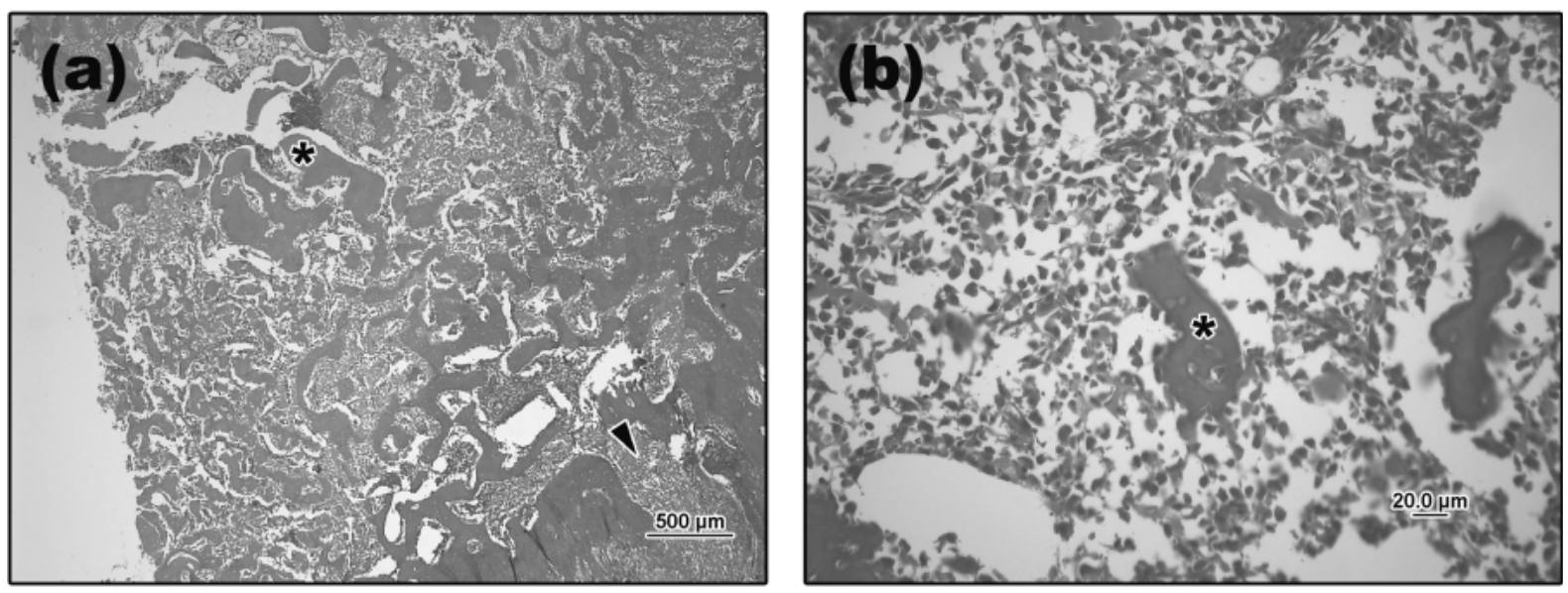

Fig. 1. Histopathological examination of the amputated left forelimb. Neoplastic cells are invading the bone tissues (black arrow head). A large amount of osteoid production (*) is seen among neoplastic osteoblasts. H\&E stain.
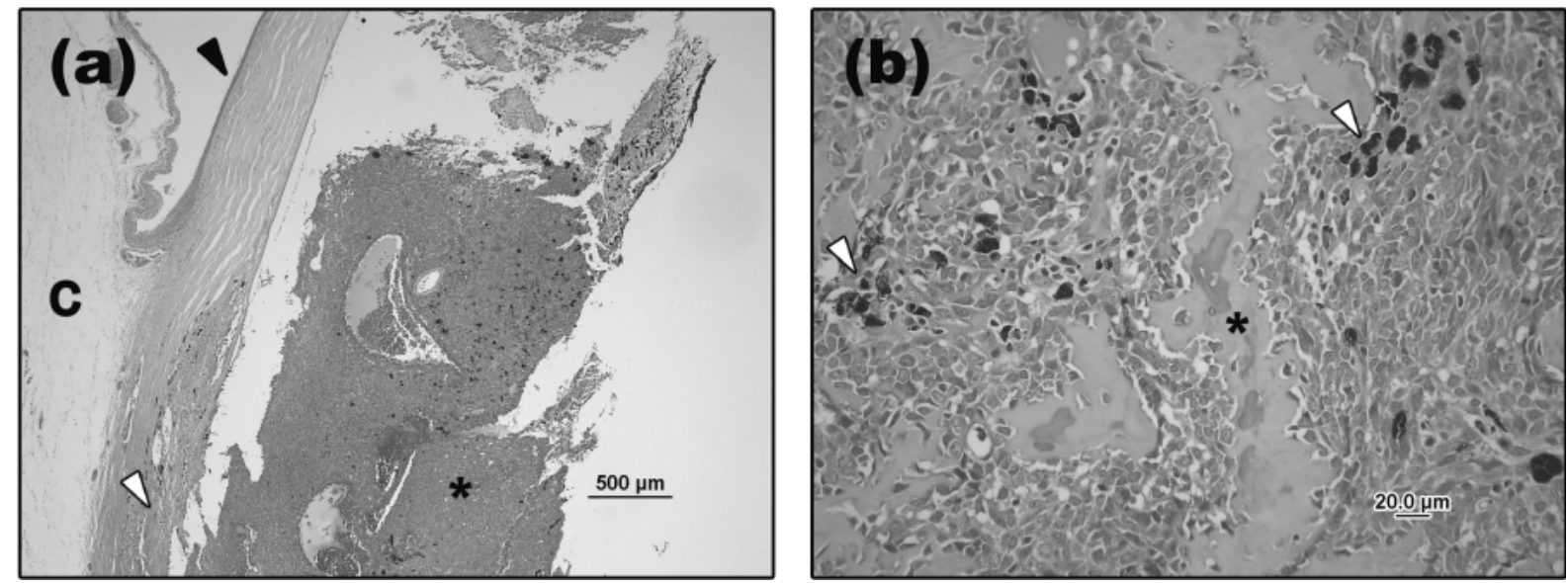

Fig. 2. Histopathological examination of the enucleated right eye. (a) The neoplastic tissue is arising from the iris $\left({ }^{*}\right)$. Normal conjunctiva (C), cornea (black arrow head) and sclera (white arrow head) are seen. H\&E stain. (b) Some melanocytes are seen (white arrow head). Highly pleomorphic neoplastic osteoblasts, surrounding the areas of eosinophilic osteoid differentiation $(*)$, are seen. H\&E stain.

submitted to another histopathologist $(\mathrm{RD})$ for a second opinion. The glass slides which were used for the first histopathological examination were evaluated, as well as new sections from the paraffin embedded block. The tumor which arose from the iris, with extension into deep aspects of nearby sclera, was an expansile, well-demarcated mass comprised of large, dense sheets of moderately pleomorphic neoplastic polygonal osteoblasts. There were moderate anisocytosis and anisokaryosis in this population with few binucleate tumor cells noted. Neoplastic osteoblasts surround several small to medium-sized, irregular islands of newly-formed bone and osteoid, which are not usually seen in normal eyes or in eyes with malignant melanoma. Some free melanin-like granules containing cells were observed, as were melanophages; however, melanin could not be visualized in tumor cells of hematoxylin and eosin (H\&E) stain sections (Fig. 2). Immunohistochemical examination revealed that these neoplastic cells were negative for Melan$\mathrm{A}$ and S-100 protein staining (Fig. 3). Subsequently, the eye was definitively diagnosed as having metastatic osteosarcoma in the anterior chamber.

Diagnosis of melanoma is sometimes difficult due to the wide variety of cell morphology of melanin cells and because of occasional invisibility of melanin granules in tumor cells. Moreover, because there are some case reports of malignant melanoma with osteoid formation, even though they are rare, light microscopic examination of glass slides with only regular stain such as H\&E stain is sometimes insufficient $[2,12,15,16]$. The existence of osteoid in the eye, which the first histopathologist did not mention, strongly suggests that these cells are osteosarcoma which metastasized from the primary site. However, in order to rule out the possibility of malignant melanoma with osteoid formation, immunohistochemical examination was also per- 

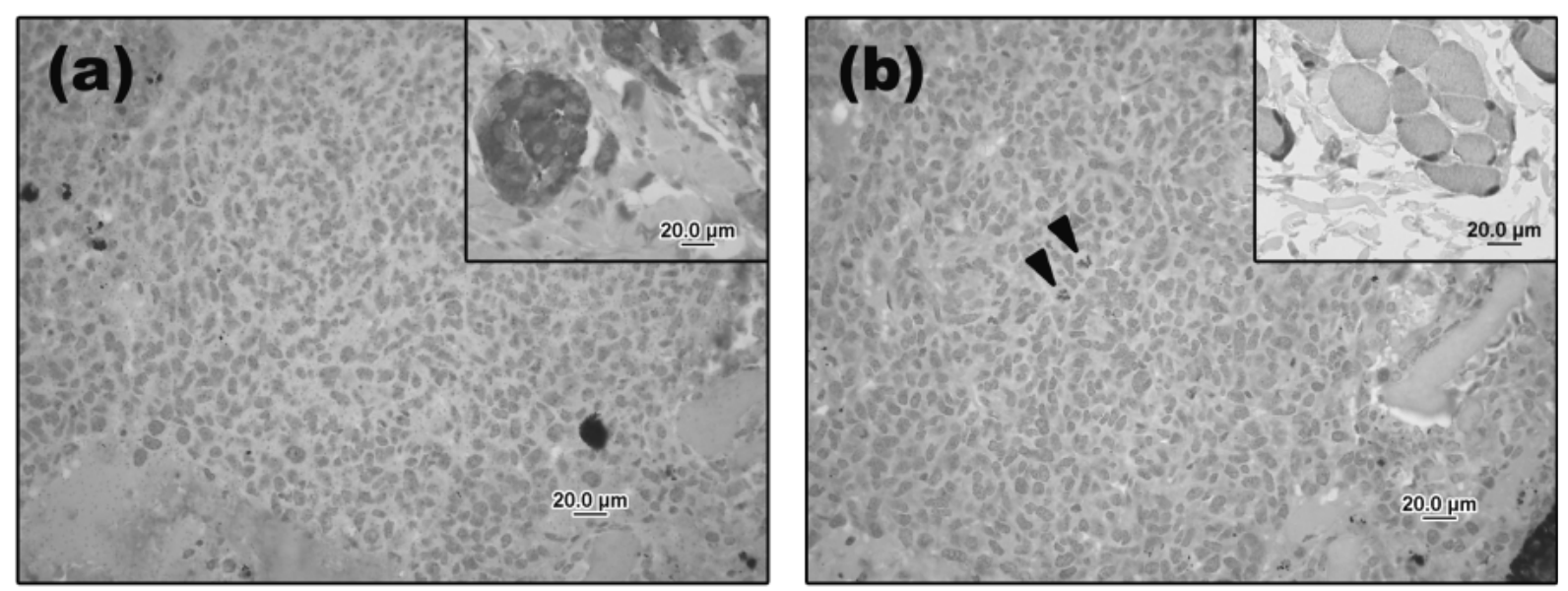

.Fig. 3. Immunohistochemical examination of the enucleated right eye. Neoplastic cells are negative for (a) Melan-A and (b) S-100 protein stain. Mitotic figures are also seen (black arrow head). In both figures, positive controls are shown in the insets.

formed. Immunohistochemical stain for Melan-A and S100 protein are useful for detecting neoplastic melanocytes, especially when they are amelanotic [8]. According to previous reports, osteoid-forming neoplastic melanocytes are always positive for Melan-A and S-100 protein [2, 12, 15, 16]. In our case, the intraocular neoplastic cells which surrounded the osteoid were negative for these stains. Considering this point and the past history of appendicular osteosarcoma, we can assert that the previous diagnosis of intraocular malignant melanoma was incorrect and that a diagnosis of metastatic osteosarcoma to the anterior chamber is appropriate.

In dogs, tumors of the eye are not very common [4]. Many different kinds of secondary intraocular tumors are reported, and lymphoma is the most common secondary intraocular tumor $[4,7,13]$. Others include hemangiosarcoma, cutaneous and oral melanoma, seminoma, transmissible venereal tumor, transitional cell carcinoma of the urinary bladder, fibrosarcoma, and pheochromocytoma [4, $7,13]$. In human medicine, breast carcinoma and lung carcinoma are the common tumors which metastasize to the eye [20], and cases of secondary orbital and choroidal osteosarcoma were also been reported $[1,10,11,14,21]$. However, these reports of secondary orbital osteosarcoma are not intraocular neoplasia, but retrobulbar cases $[1,11,14]$. In dogs, just one case of metastatic choroidal osteosarcoma and some cases of primary intraocular osteosarcoma have been reported by several authors $[6,9,19,22]$, but reports of canine secondary osteosarcoma in the anterior chamber do not exist.

In cats, post-traumatic sarcomas of the eye have been reported $[4,13]$. These tumors are thought to have been induced by trauma to the eye, and cats' eyes in particular are thought to be prone to developing invasive sarcoma $[4,13]$. However, the dog in this report did not have any past history of eye trauma, making it unlikely that osteosarcoma in the eye was induced by trauma.

There are several reports about side effects related to carboplatin administration [17]. In human medicine, a case which developed a secondary tumor following carboplatincontaining multi-drug chemotherapy has been reported [18]. However no reports in dogs exist, and the relationship between intravenously administered carboplatin and intraocular secondary osteosarcoma in this case is unknown.

In this dog, the clinical course was quite unique. The most common metastasis site of osteosarcoma is the lung parenchyma, and other sites include bone, spleen, and other soft tissues [3]. In the only report of canine metastatic choroidal osteosarcoma, the ocular lesion was found at the same time that the lung metastases were found [19]. However, in this dog, the first metastasis which was clinically detectable was in the right eye, and after enucleation he was doing very well until lung metastasis was found by radiograph. Even though computed tomography examination is thought more sensitive than conventional radiographic examination for detecting lung and other organ metastasis [5], it is not as easily available to many clinicians as conventional radiographic examination due to its accessibility and higher cost.

At the time of writing, there have been no reports about dogs with appendicular osteosarcoma that first metastasized to the anterior chamber of the eye during carboplatin chemotherapy and which were clinically doing well until lung metastasis was detected. Careful physical and ophthalmic examination may reveal more cases with osteosarcoma metastasis to the eyes at early stages, and this may lead to further understandings about metastasis to the eyes, as well as improving the patients' quality of life.

\section{REFERENCES}

1. Attili, S. V. S., Jain, A., Saini, K. V., Batra, U., Babu, G. K., Sajeevan, K. V. and Biswas, S. 2007. Orbital metastasis: a rare presentation of osteosarcoma. Int. Ophthalmol. 23: Epub ahead of print.

2. Chenier, S. and Dore, M. 1999. Oral malignant melanoma with osteoid formation in a dog. Vet. Pathol. 36: 74-76.

3. Dernell, W. S., Ehrhart, N. P., Straw, R. C. and Vail, D. M. 
2007. Tumors of the skeletal system. pp. 540-582. In: Small Animal Clinical Oncology. 4th ed. (Withrow, S. J. and Vail, D. M. eds.), W. B. Saunders, Philadelphia.

4. Dubielzig, R. R. 1990. Ocular neoplasia in small animals. Vet. Clin. North Am. Small Anim. Pract. 20: 837-848.

5. Forrest, L. J. 2007. Diagnostic imaging in oncology. pp. 97111. In: Small Animal Clinical Oncology. 4th ed. (Withrow, S. J. and Vail, D. M. eds.), W. B. Saunders, Philadelphia.

6. Heath, S., Rankin, A. J. and Dubielzig, R. R. 2003. Primary ocular osteosarcoma in a dog. Vet. Ophthalmol. 6: 85-87.

7. Hendrix, D. V. 2007. Diseases and surgery of the canine anterior uvea. pp. 812-858. In: Veterinary Ophthalmology, 4th ed. (Gelatt, K. N. ed.), Blackwell Publishing, Iowa.

8. Koenig, A., Wojcieszyn, J., Weeks, B. R. and Modiano, J. F. 2001. Expression of S100a, Vimentin, NSE, and Melan A/ MART-1 in seven canine melanoma cell lines and twenty-nine retrospective cases of canine melanoma. Vet. Pathol. 38: 427435.

9. Langenbach, A., Anderson, M. A., Dambach, D. M., Sorenmo, K. U. and Shofer, F. D. 1998. Extraskeletal osteosarcomas in dogs: a retrospective study of 169 cases (1986-1996). J. Am. Anim. Hosp. Assoc. 34: 113-120.

10. Lees, V. T. 1947. A case of metastatic osteosarcoma in the choroid. Br. J. Ophthalmol. 31: 713-716.

11. Lin, P. Y., Chen, W. M., Hsieh, Y. L., Chen, W. Y. and Chen, T. H. 2005. Orbital metastatic osteosarcoma J. Chin. Med. Assoc. 68: 286-289.

12. Lucas, D. R., Tazelaar, H. D., Unni, K. K., Wold, L. E., Okada, K., Dimarzio, D. J. Jr. and Rolfe, B. 1993. Osteogenic melanoma. a rare variant of malignant melanoma. Am. J. Surg. Pathol. 17: 400-409.
13. Miller, P. E. and Dubielzig, R. R. 2007. Ocular tumors. pp. 686-698. In: Small Animal Clinical Oncology. 4th ed. (Withrow, S. J. and Vail, D. M. eds.), W. B. Saunders, Philadelphia.

14. Misra, A., Misra, S., Chaturvedi, A. and Srivastava, P. K. 2001. Osteosarcoma with metastasis to orbit. Br. J. Ophthalmol. 85: 1387-1388.

15. Moreno, A., Lamarca, J., Martinez, R. and Guix, M. 1986. Osteoid and bone formation in desmoplastic malignant melanoma. J. Cutan. Pathol. 13: 128-134.

16. Oyamada, T., Tanaka, H., Park, C. H., Ueki, H., Komiya, T. and Arai, S. 2007. Pathology of canine oral malignant melanoma with cartilage and/or osteoid formation. J. Vet. Med. Sci. 69: $1155-1161$.

17. Plumb, D. C. 2005. Carboplatin. pp. 117-119. In: Plumb's veterinary drug handbook 5 th ed. (Plumb, D. C. ed.), Blackwell Publishing, Iowa.

18. Postovsky, S., Vlodavsky, E., Eran, A., Guilburd, J. and Ben Arush, M. W. 2007. Secondary glioblastoma multiforme after treatment for primary choroids plexus carcinoma in childhood. J. Pediatr. Hematol. Oncol. 29: 248-252.

19. Render, J. A., Carlton, W. W., Vestre, W. A. and Hoerr, F. S. 1982. Osteosarcoma metastatic to the globes in a dog. Vet Pathol. 19: 323-326.

20. Shields, C. L., Shields, J. A., Gross, N. E., Schwartz, G. P. and Lally, S. E. 1997. Survey of 520 eyes with uveal metastases. Ophthalmology 104: 1265-1276.

21. Spaulding, A. G. and Woodfin, M. C. Jr. 1968. Osteogenic sarcoma metastatic to the choroid. Arch. Ophthalmol. 80: 84-86.

22. van de Sandt, R. R. O. M., Boeve, M. H., Stades, F. C., Kik, M. J. L. and Kirpensteijn, J. 2004. Intraocular osteosarcoma in a dog. J. Small Anim. Pract. 45: 372-374. 